\title{
PARTICIPACIÓN CIUDADANA EN LA CONTRATACION ESTATAL COMO MECANISMO DE CONTROL SOCIAL EN EL DISTRITO DE CARTAGENA ${ }^{1}$
}

\section{Citizen participation in government contracting as social control mechanism in the district of Cartagena}

\author{
Autores: Milton Buelvas Mendoza ${ }^{2}$ \\ Estudiantes asistentes: \\ Martha Bermúdez Castellar, Isela Pérez Jiménez, Angie Sierra Vanegas ${ }^{3}$
}

Correspondencia: milton.buelvas@curnvirtual.edu.co - myenbume@gmail.com.

\begin{abstract}
RESUMEN
En el presente artículo de investigación se abordará la participación ciudadana en la contratación Estatal desde una visión, de lo que debe entenderse por control social, formación ciudadana, sociedad civil, cultura política y mecanismos de intervención de la comunidad. Lo anterior nos lleva a emprender uno de los enfoques metodológicos más interesantes para abordar un intento de explicar las contradicciones y desajustes de nuestro sistema de Contratación Estatal, dentro de un marco en el que los ciudadanos pueden participar en las decisiones y actividades del poder público.
\end{abstract}

\footnotetext{
${ }^{1}$ Este artículo se deriva del proyecto de investigación titulado “¿Han sido eficaces y efectivos los controles aplicados a los procesos de contratación estatal en el sector descentralizado de Cartagena D.T. y C., durante el periodo 2008-2011'?”, investigador principal: Milton Buelvas Mendoza; adelantado por el grupo de investigación de Derecho Público de la facultad de ciencias sociales y humanas - corporación universitaria Rafael Núñez. Este proyecto inicio el 1 de Abril de 2013.

${ }^{2}$ Abogado. Especialista en Seguridad Social y en Derecho Administrativo. Líder del Grupo Derecho Público de la facultad de ciencias sociales y humanas - Corporación Universitaria Rafael Núñez. Línea de investigación DESC - Eje de Gobernabilidad Democrática.

${ }^{3}$ Estudiantes del programa de derecho de la Corporación Universitaria Rafael Núñez, sede Cartagena, Investigadores Auxiliar del grupo de Derecho Público de la facultad de ciencias sociales y humanas. Línea de investigación DESC - Eje de Gobernabilidad Democrática. e-mail: asierrav13@curn.edu.co
} 
Vol.7, No 1. Diciembre de 2015 pp. $56-73$

Bajo esta perspectiva se analiza si han sido ejercidos los mecanismos de participación ciudadana en el distrito de Cartagena durante los periodos 2008-2011 para la inspección, vigilancia y decisiones que ayudan a resolver los problemas que se presentan en la contratación Estatal.

\title{
Palabras claves
}

Control social, formación ciudadana, participación, sociedad.

\begin{abstract}
In this research article we will study the Citizen participation in government procurement will be addressed from a perspective of what is meant by social control, civic education, civil society, political culture and mechanisms for community involvement. This leads us to undertake one of the most interesting methodological approaches to address an attempt to explain the contradictions and imbalances in our system of Government Contracting, within a framework in which citizens can participate in decisions and activities of government.
\end{abstract}

From this perspective we analyze whether the mechanisms have been exercised for citizen participation in the district of Cartagena during 2008-2011 periods for inspection, monitoring and decisions that help solve the problems that arise in government contracting.

\section{Key words}

Social control, citizen training, participation, partnership.

\section{INTRODUCCIÓN}

En los últimos años ha incrementado el nivel de percepción de los colombianos sobre la falta de aplicación de los mecanismos de control social en la contratación Estatal, de esta forma se entienden que existen herramientas como la participación ciudadana utilizada para fortalecer la gestión en contratos del Estado.

Uno de los mecanismos de control en la contratación Estatal es la participación ciudadana que ha tenido auge en los últimos años como lucha frente a las 
Vol.7, No 1. Diciembre de 2015 pp. $56-73$

conductas indebidas de la administración Estatal, la idea de involucrar a la ciudadanía es asegurar el control político por parte del Estado y el control social como alternativa a la coerción, para procurar la gobernabilidad y democracia.

La participación ciudadana, asume el rol de gobernanza, que alude a la responsabilidad de vincular al Estado y a la sociedad de hacer parte de la administración pública. En otras palabras la gobernanza puede entenderse como "el proceso mediante el cual una sociedad define sus objetivos estructurales y coyunturales de convivencia y define la forma de organizarse para realizarlos, de modo que los propósitos sociales de sociedad se vuelven hechos sociales, es decir, la gobernanza es una acción colectiva que por un lado define los fines o propósitos de la acción (componente intencional) y, por otro lado, define los medios idóneos para lograr los resultados propuestos" (componente teleológico causal)(Aguilar,2005, p. 10).

Asimismo, el Estado no es el único responsable de combatir las conductas indebidas en la contratación Estatal sino que además la sociedad civil a través de los mecanismos de control debe formular e implementar normas o políticas en la lucha contra este flagelo, teniendo así que la ciudadanía esté informada acerca de las dimensiones de la corrupción y los instrumentos disponibles para controlarla. Además de los controles gubernamentales, es necesario por parte del Estado incluir a la sociedad civil para la campaña anticorrupción, fortaleciendo una correcta administración.

Por consiguiente, la formulación de esta investigación es, "la participacion ciudadana en la contratación estatal como mecanismo de control en el distrito de Cartagena", producto de la investigación si ¿Han sido eficaces y efectivos los controles aplicados a los procesos de contratación estatal en el sector descentralizado de Cartagena D.T. y C., durante el período 2008-2011?, y de allí necesariamente se desprendieron los siguientes interrogantes ¿Cómo alcanzar una Gobernabilidad que no se soporte en fenómenos irregulares o de corrupción 
Vol.7, No 1. Diciembre de 2015 pp. $56-73$

sino en una cultura política basada en sanas prácticas de contratación estatal? y ¿Qué relación existe entre las exigencias del grupo social, político o económico al

que pertenece un individuo y la realización de prácticas ilegales por parte de este?.

\section{MÉTODOS.}

El enfoque metodológico que se quiere abordar, su fundamentación epistemológica, y su carácter Socio jurídica, ubica esta propuesta bajo el enfoque de Tipo Cualitativo, y tiende a ser de orden exploratorio, orientándose a estructuras teóricas y fenomenológicas, teniendo como área de trabajo el distrito de Cartagena.

Además, por su naturaleza, y siguiendo la tipología de investigaciones expuestas por Jaime Giraldo Ángel, al campo de la investigación jurídica propiamente dicha, en su modalidad de investigación jurídica básica., corresponde al método bibliográfico, el cual es usado para localizar, clasificar y evaluar informaciones, ideas, conceptos, posturas filosóficas, culturales, jurídicas, políticas, etc., consignadas en tiempos pasados o con anterioridad a la presente investigación

\section{Materiales e instrumentos de recolección de la información.}

Dado que el diseño metodológico es de carácter bibliográfico, se pretende recurrir en consecuencia a las fuentes secundarias que nutran y den contenido a la presente investigación, indagando en textos jurídicos, históricos, epistemológicos y filosóficos, buscando la información necesaria y pertinente que ayude a la solución del presente problema de investigación. 


\section{Plan de análisis de los datos.}

La comprensión, el análisis y la producción de textos jurídicos y filosóficos, más específicamente hace relación a que se den en esta investigación tres tipos de procesos:

a) El intra-textual: se refiere a determinar las estructuras semánticas y sintácticas, y además de manejar el léxico jurídico, y las estrategias que dan coherencia y cohesión a los mismos;

b) El inter-textual: se refiere a que se pueda reconocer las relaciones existentes entre el texto y otros textos, entre la jurisprudencia y la doctrina, o entre diferentes doctrinantés o jurisconsultos;

c) Extra-textual: se refiere a que se pueda reconstruir, con el fin de alumbrar el sentido de la norma o del texto jurídico, el contexto o situación de comunicación en que se producen o aparecen los textos o las normas jurídica.

\section{Unidad de análisis.}

Esta Investigación, en la medida en que es exploratoria, trabajará con las siguientes unidades de análisis:

Actores Académicos: La Academia es un actor que históricamente ha sido muy activa en los mecanismos de control de la participación ciudadana como lucha de las conductas indebidas en Cartagena. No obstante, para efectos de esta investigación solo se tomará como Unidades de Análisis una muestra de expertos sacados de las Universidades y centros de pensamiento e investigación ubicados en la ciudad de Cartagena.

Sociedad Civil: La sociedad y los líderes de los grupos sociales juegan un papel importante en cuanto a los procesos de vigilancia, control y sanción social de las conductas indebidas en materia de contratación estatal. Para esta investigación es 
importante consultar la visión, la perspectiva de sectores representativos de la Sociedad Civil del distrito de Cartagena, mediante la aplicación de Entrevistas Estructuradas, de tal manera que se pueda realizar un posterior análisis de la información.

\section{RESULTADOS Y DISCUSIÓN.}

\section{Control Fiscal Y Control Social}

El control fiscal es una función pública ejercida por la Contraloría General de la República, que vigila la gestión fiscal de la administración estatal, de los particulares y entidades que manejen bienes o fondos públicos. Dicho control lo puede ejercer de manera posterior y selectiva de acuerdo con los procedimientos, sistemas y principios que la normatividad establezca.

Con la promulgación de la Constitución Política de 1991 entró en vigencia el nuevo marco constitucional sobre el cual se estructuró el control fiscal en Colombia. Por expreso mandato de la Carta, la vigilancia de la gestión fiscal del Estado incluye el ejercicio de un control financiero, de gestión y de resultados, fundado en la eficiencia, la economía, la equidad y la valoración de los costos ambientales. y son atribuciones del Contralor General de la República "prescribir los métodos y la forma de rendir cuentas los responsables del manejo de fondos o bienes de la Nación e indicar los criterios de evaluación financiera, operativa y de resultados que deberán seguirse, así como revisar y fenecer las cuentas que deben llevar los responsables del erario y determinar el grado de eficiencia, eficacia y economía con que hayan obrado", de conformidad con lo dispuesto en los numerales 1 y 2 del artículo 268 de la Constitución Política.

Es posible que el control fiscal haya disminuido los casos de corrupción, o el ejercicio de sus funciones haya descubierto muchos casos, sobre todo en el nivel 
Vol.7, No 1. Diciembre de 2015 pp. 56 -73

nacional. Por otra parte, el control social es una actividad, una acción que busca la vinculación directa de los ciudadanos con el ejercicio de lo público, con el propósito de que la gestión pública cumpla lo que está establecido en la Constitución de 1991 cuando señala en el artículo 2, que entre los fines esenciales del Estado está "facilitar la participación de todos en las decisiones que los afectan y en la vida económica, política, administrativa y cultural de la Nación”.

El espíritu que anima y rige el control social es el ejercicio consciente, ininterrumpido y ético en el que se comprometen los ciudadanos con el fin de hacerle seguimiento a la gestión del Estado, para que las instituciones cumplan con sus objetivos y metas. "En Colombia son varios los actores del control social: ciudadanos y ciudadanas de manera individual; comunidades, organizaciones no gubernamentales (ONG), cámaras de comercio, universidades y asociaciones gremiales que, por su iniciativa, llevan a cabo veedurías ciudadanas u otras formas inéditas e informales de control social a la administración pública; órganos o instancias institucionalizadas o formalizadas de participación ciudadana de tipo territorial, sectorial y exclusivo de control social convocados por el Estado; usuarios de servicios públicos domiciliarios y de servicios de salud; y medios de comunicación" (Corporación Transparencia por Colombia, capítulo Transparencia Internacional, 2011). Pero hasta el momento son más las posibilidades que tiene el control social en su cobertura y objetivos que en su realidad.

El fin del control social en Colombia va desde la prevención hasta la acusación y sanción. Y tiene unos mecanismos que los ayudaría a cumplir sus objetivos:

- Hacer visible y difundir al público información oficial.

- Recomendar medidas para hacer más transparentes la información, las reglas de actuación y/o la toma de decisiones.

- Denunciar o demandar judicial, disciplinaria o fiscalmente, con fines de corrección y/o de sanción.

- $\quad$ Criticar con fines de corrección o de sanción social. 
Vol.7, No 1. Diciembre de 2015 pp. $56-73$

- Hacer seguimiento para verificar cumplimiento de actividades con fines de prevención, corrección o información al público.

- Hacer seguimiento al cumplimiento de compromisos y metas de las autoridades, con fines de debate, crítica o corrección (Corporación Transparencia por Colombia, capítulo Transparencia Internacional, 2011).

Sin embargo, falta mucho por afinar sobre este tipo de control que puede ser seducido por los agentes de la corrupción. Pero sin duda, es muy interesante insistir en la realidad sobre los beneficios del control social, como ocurrió en Cartagena, y en la Alcaldía Distrital de Cartagena de Indias, con el Plan de Desarrollo 2008 -2011, donde se diseñó una estrategia denominada la Administración al Servicio de Todos y Todas, cuyo objetivo fue dotar de una estructura orgánica adecuada a la Administración Distrital, logrando desarrollar aplicaciones como la publicación Servicios SIGOB (Sistema de Información de la Gestión para la Gobernabilidad) en Internet, el mejoramiento de la infraestructura y capacidad tecnológica de la Alcaldía, la entrega de información al ciudadano y la transparencia y gobernabilidad. Esto posibilitó entregar información oportuna y veraz a la ciudadanía, promoviendo procesos transparentes, además de articular los diversos procedimientos que se integran en la gestión municipal (PNUD, 2009), y se desarrollaron actividades con la sociedad para construir de manera participativa políticas públicas en beneficio de todos.

\section{Formación Ciudadana, Sociedad Civil Y Cultura Política}

Debería estar fuera de todo debate la importancia de los procesos de formación ciudadana para una Nación que quiera profundizar su democracia, alcanzar altos niveles de calidad de vida y construir una sociedad ética, donde, por ejemplo, la corrupción no tenga cabida.

Es formación ciudadana el conjunto de acciones que buscan el desarrollo local y la democracia participativa, a través de la sociedad, y que posibilita acceder e incidir 
Vol.7, No 1. Diciembre de 2015 pp. $56-73$

en las decisiones gubernamentales en los ámbitos nacional, regional, departamental, municipal, barrial, sin hacer parte de ningún partido político o de la administración pública (Giner, 1998).

La formación ciudadana implica aprender y aprehender a superar los conflictos, las inequidades sociales y económicas, construir espacios para la participación ciudadana, enfrentar los altos indicadores de corrupción, pero también encontrar ambientes armoniosos institucionales, estatales y civiles, donde las competencias ciudadanas participen de estos objetivos e involucren a la sociedad civil en general. "El ciudadano se construye para que la sociedad se transforme y ese proceso de construcción ciudadana es un proceso de transformación social" (Cepeda, 2004). No obstante, el asunto no es sencillo. En el devenir histórico de la humanidad, las relaciones entre sí son indispensables para vivir. Pero surgen grupos de interés que luchan por unos objetivos concretos que no necesariamente benefician a la sociedad como tal. Unos pretenderán apropiarse del Estado, del erario, de la junta directiva de una entidad, o de la acción comunal. En ese proceso de tensiones entre los grupos surgen, en muchos casos, los fenómenos de corrupción.

Si existen altos estándares de competencias ciudadanas, estas pueden convertirse en barreras a la entrada y la salida, para controlar los fenómenos de corrupción. La formación ciudadana se desarrolla ejerciéndola, poniéndola en práctica y arrojando resultados que la estimulen a su propio crecimiento (Mockus, 2004).

El concepto de competencias ciudadanas, herramienta necesaria para ejercer la ciudadanía, se desarrolla aún más cuando en la puesta en marcha de esa habilidad se resuelven los conflictos de intereses de forma pacífica, o logra desarticular actos de corrupción que quedan solo en la tentativa. 
Vol.7, No 1. Diciembre de 2015 pp. 56 -73

Los procesos de formación ciudadana también buscan los consensos que solo pueden obtenerse “... si todos participan libremente: no podemos esperar que surja el consentimiento a menos que todos los afectados puedan aceptar libremente las consecuencias y afectos secundarios que pueda tener para la satisfacción de los intereses de cada persona" (Habermas, 1985).

Una de las razones que a los actores de la corrupción les inquieta es la participación ciudadana, con alta formación en sus deberes y derechos, y por la cual tratan de ganársela para sus propósitos, es por ese proceso de formación que cambia rotundamente los comportamientos fácticos y éticos de la comunidad: "La ciudadanía es un mínimo de humanidad compartida. Cuando decimos que alguien es ciudadano pensamos en aquel que respeta unos mínimos, que genera una confianza básica. Ser ciudadano es respetar los derechos de los demás. El núcleo central para ser ciudadano es, entonces, pensar en el otro. Se basa en tener claro que siempre hay un otro, y tener presente no solo al otro que está cerca y con quien sabemos que vamos a relacionarnos directamente, sino también considerar al otro más remoto, al ser humano aparentemente más lejano -al desconocido, por ejemplo- o a quien hará parte de las futuras generaciones (...) pero el ciudadano también se define por su relación con el Estado. Uno es ciudadano de un país específico con unas normas establecidas por un Estado específico. Cuando se habla de las consecuencias, un ciudadano no solo mira las consecuencias para unos, sino para todos" (Mockus y Corzo, 2003).

El concepto de ciudadanía, la relación de los miembros de la sociedad con el Estado, exigir sus derechos constitucionales, ejercer los derechos humanos, mediante la acción ciudadana, producto de esa formación en sus derechos y deberes, se convierte en un instrumento que puede llevar a la sociedad a otros estadios de desarrollo de ella misma y lograr que el Estado cumpla su rol, y el erario solo cumpla las funciones para lo que fue creado. 
Vol.7, No 1. Diciembre de 2015 pp. 56 -73

Pero todos estos conceptos quedarían debilitados sin hacer referencia a la cultura política, entendida como el conjunto de creencias y valores compartidos, referentes a la vida en sociedad y al rol de las actividades políticas en la conservación y la orientación de la cohesión social; conjunto de actitudes fundamentales que permiten el ajuste mutuo de los comportamientos o la aceptación de actos de autoridad que tienden a imponer ese ajuste (Lagroye, 1973).

Si la formación ciudadana estuviese acompañada de una cultura política, en los términos aquí expuestos, estaríamos en la posibilidad de tener un Estado social de derecho, pero también en la realidad donde el ciudadano tiene una participación con alta calidad y la corrupción llegaría a ser asunto del pasado.

Un elemento importante de toda democracia es garantizar que los integrantes de una sociedad puedan participar de forma real en las decisiones colectivas. Y la Constitución así lo deja consignado cuando define a Colombia como una República democrática y participativa. Esa forma de participación es mediante representantes o de manera directa. La participación directa está consagrada en el artículo 40 de la Carta, en las formas de participación de los pobladores en la vigilancia y fiscalización de la gestión pública, en la participación en la función administrativa, en la administración de justicia por particulares, en los mecanismos judiciales para la protección de intereses públicos, entre otros. Y esta participación es "un elemento de importancia estructural para el ordenamiento constitucional colombiano; tanto así que, de conformidad con el preámbulo y los artículos 1 y 2 de la Carta, es uno de los principios fundantes del Estado y, simultáneamente, uno de los fines esenciales hacia los cuales se debe orientar su actividad" (Sentencia C-169/01). 
Los planes de desarrollo de varios municipios, entre ellos, Por una sola Cartagena, (2008-2011), fueron producto de la participación organizada de comunidades que con sus opiniones y recomendaciones lo enriquecieron.

\section{Sociedad Civil}

Es conveniente diferenciar entre la sociedad política, constituida por los partidos, por ejemplo, y la sociedad civil, como necesaria para la existencia de la democracia. Esa sociedad civil está compuesta por: las instituciones que se encuentran en la defensa de los derechos civiles individuales y políticos de los ciudadanos, que impulsan su libre asociación y que se agrupan para defenderse de los atropellos del poder y el mercado, y, por otra parte, por los movimientos sociales que propugnan por nuevas demandas para satisfacer sus necesidades colectivas y que están pendientes del cumplimiento de los derechos que las normas le han consagrado (Habermas, 2008).

No se encuentra el Estado por encima del poder ciudadano ni de la sociedad civil, la cual puede desobedecer cuando los mandatos de ese Estado van en contravía de los intereses de esa sociedad, no solo desde lo económico sino desde lo ético (Thoreau, 1849).

Por su parte, Alexis de Tocqueville considera que la sociedad civil es el conjunto de organizaciones cívicas que sirven de puente entre los individuos y el Estado en la consecución de sus demandas colectivas (Aramayo, 2011).

La sociedad civil, con sus precariedades, se ha movilizado a través de nuestra historia; modernamente, el movimiento que se conoció como el de la Séptima Papeleta fue una manifestación de la sociedad civil, pero desde la época de Manuela Beltrán y de Policarpa Salavarrieta, la sociedad civil se expresó y logró conformarse en movimientos sociales. 


\section{Mecanismos de participación ciudadana:}

Antes de mencionar cuales son los mecanismos de control utilizados por la sociedad civil para combatir el flagelo de la corrupción es destacar" el importante rol que puede jugar la sociedad en evaluar el desempeño o calidad de los servicios del gobierno y advierte que el control social será efectiva siempre que sea fundada en una relación constructiva entre la sociedad y el Estado. Indica, además, que ante la insuficiencia de la disponibilidad de información (transparencia) para garantizarla rendición de cuentas, es necesario que los gobiernos estimulen la participación ciudadana e institucionalicen mecanismos que la hagan viable" (Ackerman,2004, p.30).

Sin embargo, el control de la sociedad civil como mecanismo de lucha contra la corrupción es débil, por las limitaciones intelectuales de la ciudadana en tener una participación popular buena, ya que los ciudadanos no tienen suficientes conocimientos para participar efectivamente en los procesos de toma de decisiones, de modo que la ignorancia sobre aspectos legales, financiero y técnicos son el principal obstáculo para legitimar su participación.

Los mecanismos de participación ciudadana inicia con la expedición de la constitución política de 1991, por dejar de ser una democracia representativa ha ser una participativa puesto que antes la participación de los ciudadanos en la toma de decisiones y en el seguimiento y evaluación de la gestión de los 
funcionarios y de las entidades públicas se limitaba, en el mejor de los casos, a elegir a sus representantes. No existían mecanismos ciudadanos para proponer, discutir, controlar y reclamar.

La carta magna quiso transformar esta situación introduciendo los siguientes mecanismos de control: 1. "Iniciativa popular legislativa y normativa ante las corporaciones públicas. La iniciativa popular legislativa y normativa ante las corporaciones públicas es el derecho político de un grupo de ciudadanos de presentar Proyecto de Acto Legislativo y de ley ante el Congreso de la República, de Ordenanza ante las Asambleas Departamentales, de Acuerdo ante los Concejos Municipales o Distritales y de Resolución ante las Juntas Administradoras Locales, y demás resoluciones de las corporaciones de las entidades territoriales, de acuerdo con las leyes que las reglamentan, según el caso, para que sean debatidos y posteriormente aprobados, modificados o negados por la corporación pública correspondiente.

2. Referendo Es la convocatoria que se hace al pueblo para que apruebe o rechace un proyecto de norma jurídica o derogue o no una norma ya vigente. Referendo derogatorio.

3. referendo derogatorio es el sometimiento de un acto legislativo de una ley, de una ordenanza, de un acuerdo o de una resolución local en alguna de sus partes o en su integridad, a consideración del pueblo para que éste decida si lo deroga o no.

4. Referendo aprobatorio. Un referendo aprobatorio es el sometimiento de un proyecto de acto legislativo, de una ley, de una ordenanza, de acuerdo o de una resolución local, de iniciativa popular que no haya sido adoptado por la corporación pública correspondiente, a consideración del pueblo para que éste decida si lo aprueba o lo rechaza, total o parcialmente.

5. Revocatoria del mandato. La revocatoria del mandato es un derecho político, por medio del cual los ciudadanos dan por terminado el mandato que le han conferido a un gobernador o a un alcalde. 
6. El plebiscito. El plebiscito es el pronunciamiento del pueblo convocado por el Presidente de la República, mediante el cual apoya o rechaza una determinada decisión del Ejecutivo.

7. Consulta popular. Es la institución mediante la cual, una pregunta de carácter general sobre un asunto de trascendencia nacional, departamental, municipal, distrital o local, es sometida por el Presidente de la República, el gobernador o el alcalde, según el caso, a consideración del pueblo para que éste se pronuncie formalmente al respecto. En todos los casos, la decisión del pueblo es obligatoria. Cuando la consulta se refiera a la conveniencia de convocar una asamblea constituyente las preguntas serán sometidas a consideración popular mediante ley aprobada por el Congreso de la República.

8. El cabildo abierto es la reunión pública de los concejos distritales, municipales o de las juntas administradoras locales, en la cual los habitantes pueden participar directamente con el fin de discutir asuntos de interés para la comunidad" (Ley 134 De 1994, Congreso De Colombia).

\section{CONCLUSIONES.}

1. A partir de la expedición de la constitución política de 1991 son creados los mecanismos de participación ciudadana que permiten ejercer control social en la contratación Estatal.

2. Los controles ejercidos por la sociedad civil están consagrados en la carta magna para el fortalecimiento democrático y éxito de las propuestas para las conductas indebidas de la contratación del Estado, sin embargo las instituciones estatales no fomentan los canales de participación ciudadana disponibles por mandato constitucional.

3. La escasa participación ciudadana y la baja formación ciudadana contribuye a que no exista control social sobre la contratación estatal. 
4. El desconocimiento de los mecanismos de participación ciudadana conlleva a ejercer bajo control en los procesos de contratación Estatal.

5. Se observa baja participación de la población en el control social a la contratación oficial en el sector descentralizado del Distrito de Cartagena.

6. El control social será efectivo si los ciudadanos alcanzan altos estándares de formación ciudadana para poder participar con éxito en dicho control.

7. La distribución funcional del Estado colombiano en su sector descentralizado posibilita mayor eficacia y eficiencia en la contratación estatal.

8. El sistema de controles internos y externos con que cuenta el Estado colombiano, aplicado al sector descentralizado del Distrito de Cartagena D. T. y C., reducirían los riesgos de ocurrencia de irregularidades y las prácticas de corrupción percibidas por el ciudadano en los procesos de contratación administrativa en sus diversas entidades.

9. El debilitamiento de los controles fiscales y sociales control facilita los procesos de corrupción.

\section{REFERENCIAS.}

Alcaldía Mayor del Distrito de Cartagena. Proyecto de plan de desarrollo Distrital 2008- 2011. "Por una sola Cartagena". En línea:

http://www.minambiente.gov.co/documentos/proyecto_plan_desarrollo_cartagena. pdf 03 de Abril de 2013. 5:15 p.m.

Agenda Ciudadana Por La Transparencia- Bolivar $\mathrm{Y}$ Cartagena (2011)http://www.funcicar.org/sites/default/files/archivos/agenda bolivar impres.pd f[consulta: sábado 18 de abril de 2015]

Constitución Política De Colombia. Legis Editores S.A. Bogotá. 1ª edición. 1996. 
Corporación Transparencia por Colombia. Capitulo Transparencia Internacional. La corrupción en Colombia - radiografía y retos. :http://www.transparenciacolombia.org.co/index.php?option=com_content\&view=ar ticle\&id=107\&ltemid=536[Consulta: sábado 18 de abril de 2015]

Corporación Transparencia por Colombia. Capitulo Transparencia Internacional. La corrupción en Colombia - radiografía y retos.

http://www.transparenciacolombia.org.co/index.php?option=com_content\&view=art icle\&id=107\&ltemid=536[Consulta: domingo 19 de abril de 2015]

Cortes Acero, Fernando Alexander; Herrera Díaz, Carlos Alfonso (2009). Contratación administrativa y corrupción en Colombia. Fallos de la sala de Casación Penal de la Corte Suprema de Justicia. Bogotá D.C. Escuela Superior de Administración Pública.

Documento Conpes 3186 (2002). Una política de estado para la eficiencia y transparencia en la contratación pública. Departamento Nacional de Planeación. República de Colombia. Bogotá, D.C. En línea:

http://www.colombiacompra.gov.co/sites/default/files/normativas/conpes3186.pdfR

Documento Conpes 3249 (octubre 2003). Consejo Nacional de Política Económica y Social. Política de contratación pública para un estado gerencial. República de Colombia. Departamento Nacional de Planeación. Bogotá, D.C. En línea:

https://www.contratos.gov.co/Archivos/normas/Conpes_3249.pdf

Hernández Sampieri, Roberto; Fernández-Colado, Carlos; Baptista Lucio, Pilar. Metodología de la Investigación. Cuarta Edición. McGraw Hill / Interamericana Editores, S.A. DE C.V. México, 2006.

http://cdim.esap.edu.co/BancoMedios/Documentos\%20PDF/a6853\%20\%20 contratacion\%20administrativa\%20y\%20corrupcion\%20en\%20colombia\%20(pag\% 20108\%20-\%20506\%20kb).pdf 
KEEFER, Snack, 2002, en el informe "El Conflicto, callejón con salida" - Informe Nacional de Desarrollo Humano Colombia 2003 - de las Naciones Unidas.

Ley 1474. Dicta normas orientadas a fortalecer los mecanismos de prevención, investigación y sanción de actos de corrupción y la efectividad del control de la gestión pública. (2011)

Periódico el Espectador, Destituido e inhabilitado gobernador de Bolívar Joaco Berrio (2014). http://www.elespectador.com/articulo190041-destituido-einhabilitado-gobernador-de-bolivar-joaco-berrio [consulta: viernes,28 de marzo de 2014]

Programa de las Naciones Unidas para el Desarrollo -PNUD- Colombia (2011). Evaluación externa de los resultados del proyecto 'Transparencia en la gestión pública a nivel territorial en Colombia'.

Programa de las Naciones Unidas para el Desarrollo -PNUD- Colombia (diciembre 2011). Buenas prácticas de transparencia en la gestión contractual. http://www.pnud.org.co/img_upload/33323133323161646164616461646164/gobier no/Libro\%20buenas\%20practicas.pdf[Consulta: viernes 17 de abril de 2015] 